\title{
A novel computerised quantification of thyroid vascularity in the differentiation of malignant and benign thyroid nodules
}

\author{
Seyed Babak Moosavi Toomatari ${ }^{1 A, B, C, D, E, F, G}$, Afshin Mohammadi2A,B,D,E,F, Nariman Sepehrvand ${ }^{3 A, C, D, E, F,}$, \\ Seyed Ehsan Moosavi Toomatari ${ }^{4 A, D, F}$, Mohammad Ghasemi-Rad ${ }^{5 B, D, E}$, Saber Zafar Shamspour ${ }^{6 C, D, E}$, \\ Seyfollah Rezayi ${ }^{7 B, D, E}$, Mohammadreza Toubaei ${ }^{1 B, D, E}$, Zahra Karimi Sarabi ${ }^{8 B, D, E}$
}

\author{
'Department of Surgery, Zanjan University of Medical Sciences, Zanjan, Iran \\ 2Department of Radiology, Urmia University of Medical Sciences, Urmia, Iran \\ ${ }^{3}$ Department of Medicine, University of Alberta, Edmonton, Alberta, Canada \\ ${ }^{4}$ Department of General Surgery, Tabriz University of Medical Sciences, Tabriz, Iran \\ ${ }^{5}$ Department of Radiology, Baylor College of Medicine, Houston, Texas, USA \\ ${ }^{6}$ Department of Neurosurgery, Shiraz University of Medical Sciences, Shiraz, Iran \\ 'Department of Surgery, Urmia University of Medical Sciences, Urmia, Iran \\ ${ }^{8}$ Department of Anaesthesiology, Urmia University of Medical Sciences, Urmia, Iran
}

\section{Abstract}

Purpose: Only five percent of thyroid nodules are malignant. It is important to find reliable and at the same time non-invasive methods to identify high-risk nodules. The aim of this study was to determine the diagnostic validity of a morphologic feature-oriented approach of ultrasound study for the identification of malignant thyroid nodules.

Material and methods: Seventy-one thyroid nodules in 71 consecutive patients were evaluated with both ultrasonography (US) and US-assisted fine needle aspiration biopsy (FNAB). Thyroid grey-scale and power Doppler US were performed, and a Windows-based software was designed to process power Doppler US (PDUS) images that were recorded directly by the US device. We provided a histogram graph of coloured pixels and calculated the Malignancy Index to identify the probability of malignancy for each thyroid nodule.

Results: Thirty-six nodules (50.7\%) were determined to be malignant in FNAB. Area under the receiver operating curve was 0.91 (95\% CI: 0.85-0.98) for PDUS-based malignancy index in differentiating malignant thyroid nodules from benign ones. The best cut-off point for malignancy index was determined to be 0.092 , with a sensitivity of $86.1 \%$ and specificity of $80 \%$ in identifying malignant nodules.

Conclusions: This PDUS-driven malignancy index using a contour-finding algorithm approach could accurately and reliably differentiate malignant and benign thyroid nodules. As a pre-FNAB assessment, the malignancy index may be able to reduce the number of patients with nodular thyroid disease undergoing this invasive procedure.

Key words: ultrasonography, thyroid nodule, colour mapping, malignancy.

\section{Introduction}

Thyroid nodules are the most common thyroid disease, and $4-7 \%$ of the adult population have palpable thyroid nodules [1]. Moreover, 5\% of thyroid nodules are malignant, and thyroid malignancies constitute about $1 \%$ of all cancers annually [2]. The clinical presentation differs between incidental and solitary nodules, in which cancer is

\section{Correspondence address:}

Prof. Afshin Mohammadi, Department of Radiology, Urmia University of Medical Sciences, Urmia, Iran, phone: 989143480425 ,

e-mail: Afshin.mohdi@gmail.com

Authors' contribution:

A Study design · B Data collection · C Statistical analysis · D Data interpretation · E Manuscript preparation · F Literature search · G Funds collection 
a concern and should be ruled out [3]. The key point in the evaluation of a thyroid nodule is the discrimination between the benign and malignant forms of the thyroid nodules.

There are many modalities that are used to assess the pathology of thyroid nodules, which include palpation, imaging, and biopsy. Ultrasonography is a simple, non-invasive, easily accessible, and cheap modality that is potentially useful to evaluate thyroid nodules. Several ultrasonographic characteristics such as taller-than-wide shape, marked hypoechogenicity, microcalcifications, irregular or microlobulate margins [4], and increased nodular flow visualised by Doppler ultrasound are shown to be associated with an increased risk of malignancy in nodular thyroid disease [3,5-9].

To date, ultrasound has had a limited specificity in the diagnosis and prognostication of thyroid malignancies [3,5-8]. Some authors have suggested a change in the approach of using individual ultrasonographic characteristics, suggesting new pattern recognition methods in the diagnosis of thyroid nodules. The purpose of this study was to evaluate the diagnostic accuracy of an ultrasounddriven diagnostic index for the identification of benign or malignant thyroid nodules.

\section{Material and methods}

\section{Participants}

From April 2010 to April 2013, 71 thyroid nodules from 71 consecutive patients were evaluated in the Radiology Department with both ultrasonography (US) and USassisted fine needle aspiration biopsy (FNAB). Each patient signed an informed consent form prior to participation in the study, and they were free to withdraw at any step. The study was approved by the institutional review board of the Urmia University of Medical Sciences, Urmia, Iran.

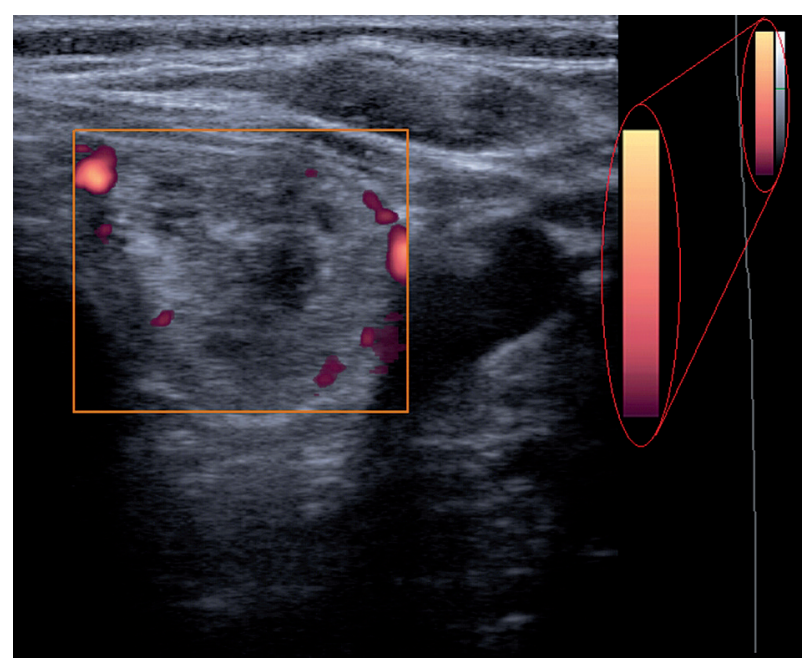

Figure 1. Ultrasonogram shows power Doppler sonography image of a thyroid nodule
A single radiologist with nine years of experience performed all the studies.

All the patients were followed up for one year, and they were re-evaluated at the end of 12 months by both ultrasound and FNAB methods. Only those patients with definitive diagnosis according to the FNAB report were included in the final analysis. Nodules were considered as benign if they showed no change in the one-year follow-up or repeated FNAB showed no change in cytological findings. The exclusion criteria (according to cytological report) were: indeterminate cytological changes, suspicious for malignancy or inadequate cytological findings.

\section{Ultrasonography}

Thyroid grey-scale and power Doppler US were performed using a Medison Accuvix V20 machine (Medison, South Korea) with a $7-12-\mathrm{MHz}$ broadband width transducer. The colour map of this device consists of 128 colours, each representing a different velocity (Figure 1).

Power Doppler ultrasonography (PDUS) was performed for all of the subjects before undergoing FNAB. We assessed the arteriole blood flow within the thyroid nodules. Power Doppler sonographies were used (pulse repetition frequency at minimum $-0.4-0.6 \mathrm{kHz}$ ) to obtain even the low blood flows. Specific settings included Doppler depth of $3 \mathrm{~cm}$; overall gain equal to $60 \mathrm{~dB}$; time gain compensation vertical and centred; power Doppler gain of 15-20 dB; and wall filter set at minimum.

\section{Fine needle aspiration biopsy}

An experienced radiologist performed ultrasound-guided FNAB in all patients with extended neck and supine position. In the first stage, after preparing a sterile field on the neck, the lesion was localised. Under ultrasound observation, until it was visible as a bright spot on the monitor, a 23-gauge needle attached to a $20-\mathrm{ml}$ syringe was inserted perpendicularly to the neck until the needle was introduced to the target thyroid nodule. This procedure was performed without local anaesthesia. On real-time monitoring, aspiration and suction were performed with to-and-from movements, and suction was released before withdrawing the needle. At least four suction attempts were made. Subsequently, needle aspiration staining with Papanicolaou and Giemsa was performed on glass slides.

The results of the thyroid cytopathology, which were assessed by an experienced thyroid pathologist, were reported at the first step as diagnostic (satisfactory: at least six groups of benign follicles, each group composed of at least 10 cells in aspirated samples) or non-diagnostic (or unsatisfactory).

Cytopathology was reported as benign (colloid nodules, adenomatous nodules, lymphocytic thyroiditis, and other), indeterminate (follicular or Hurthle cell neo- 
plasm), suspicious for malignancy, or malignant (papillary, follicular, medullary, and undifferentiated thyroid carcinoma), according to the Bethesda System for reporting thyroid cytopathology. Cytologic results were considered malignant when the specimen showed abundant cells with cytological features of cancer. Only those patients with a definitive pathological diagnosis such as benign or malignant disease were included in the study.

\section{Malignancy Index}

We designed a Windows-based software to process power Doppler US images that were recorded directly by the US device in Bitmap [BMP] format. In order to process the images, they were transferred from the US device to a desktop computer. All images had the same characteristics: width, 1024 pixels; height, 768 pixels; vertical and horizontal resolution, $72 \mathrm{dpi}$; and bit depth, 24 . The processing of images in this software consists of the four following steps (Figure 2):

Step one: Loading the power Doppler US bitmap image and extracting the border of thyroid nodule by manually drawing a region of interest; Step two: measuring total pixels, number of coloured pixels, and number of pixels for each colour in the thyroid nodule; Step three: providing the histogram graph of the coloured pixels (Figure 3) and calculating the mean and standard deviation; Step four: calculating the Malignancy Index to identify the probability of malignancy for each thyroid nodule as defined below:

The flow speed in power Doppler images is shown as colour dots (pixels). The colour spectrum consisted of 128 different colours, and each speed was designated as one colour. We then drew a histogram for each nodule, with the horizontal axis showing the speed of the flow in the nodule as showed in the power Doppler image, and the vertical axis showing the number of coloured dots or pixels corresponding to that speed. By having the histogram for each nodule, we determined the mean and standard deviation of the flow speed and calculated the malignancy index (Zahra Index) based on these numbers and other parameters that we extracted from the power Doppler images.

$$
\text { Zahra Index }=\frac{\frac{\text { Standard deviation }}{\text { Average }} \times \text { Coloured pixels }}{(\text { Total pixels })} \times 100
$$

\section{Statistical analysis}

Receiver operating characteristic (ROC) plots were used to illustrate the sensitivity versus specificity for all possible cut-off values. The area under the curve (AUC) was calculated as a measure of test accuracy to distinguish malignant tumours from benign nodules. All data analyses were performed using SPSS (Version 21.0; IBM SPSS Corporation, Chicago, IL).

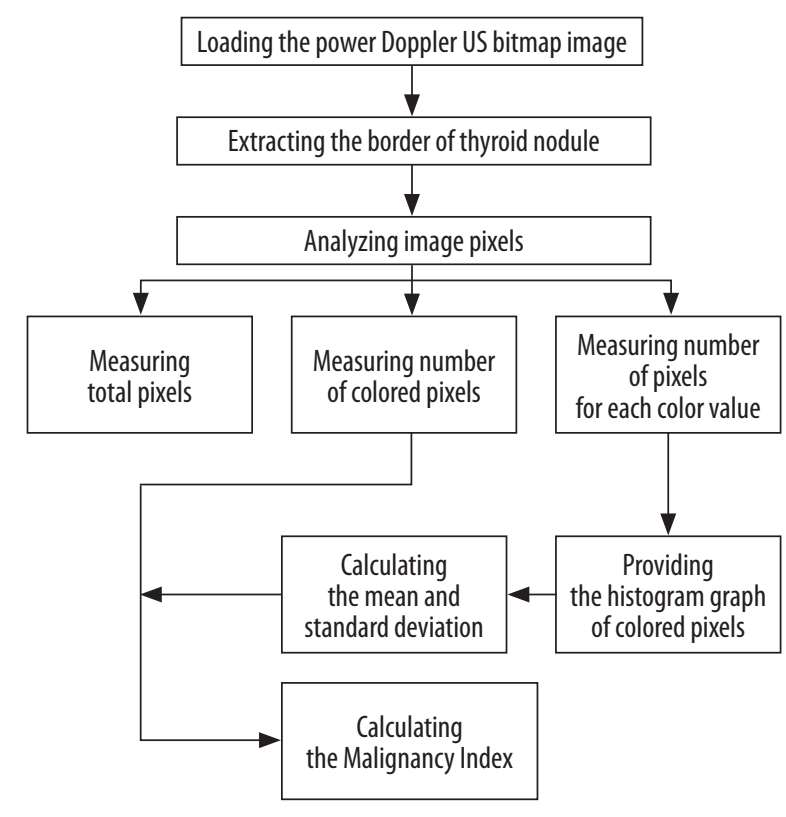

Figure 2. Simple algorithm of analysing power Doppler images

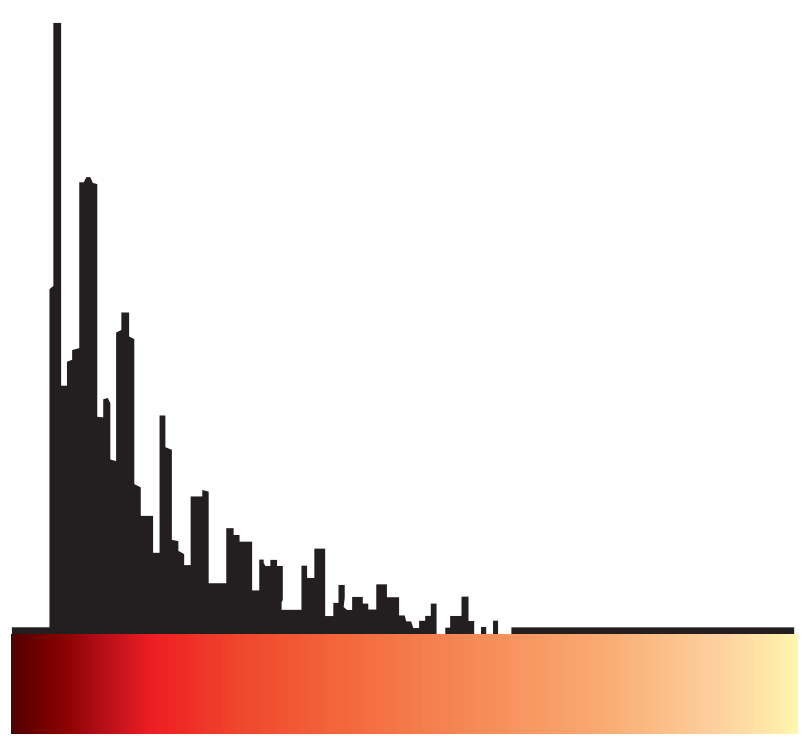

Figure 3. Histogram graph of pixel scattering for power Doppler data of a thyroid nodule

\section{Results}

Thirty-six nodules (50.7\%) were determined to be malignant via FNAB, and 35 nodules (49.3\%) were diagnosed to be benign (Table 1). ROC curve analysis found an AUC of 0.91 (95\% CI: 0.85-0.98) for PDUS-based malignancy index in distinguishing malignant tumours from the benign ones. All possible cut-off values were assessed in the ROC curve analysis (Figure 4). The best trade-off point between sensitivity and specificity was a malignancy index of 0.092 , showing a sensitivity of $86.1 \%$ (95\% CI: 70.5 $95.3 \%)$ and specificity of $80.0 \%$ (95\% CI: 63.0-91.5\%). The positive predictive value, negative predictive value, 
Table 1. Diagnostic performance of PDUS-driven malignancy index

\begin{tabular}{|c|c|c|c|c|}
\hline & & \multicolumn{2}{|c|}{ Pathology } & \multirow[b]{2}{*}{ Total } \\
\hline & & Malignant & Benign & \\
\hline \multirow{3}{*}{$\begin{array}{l}\text { Malignancy } \\
\text { index }\end{array}$} & Malignant & TP 31 & FP 7 & 38 \\
\hline & Benign & FN 5 & TN 28 & 33 \\
\hline & Total & 36 & 35 & 71 \\
\hline
\end{tabular}

FN - false negative, $\mathrm{FP}$ - false positive, TN - true negative, TP - true positive, PDUS - power Doppler ultrasonography

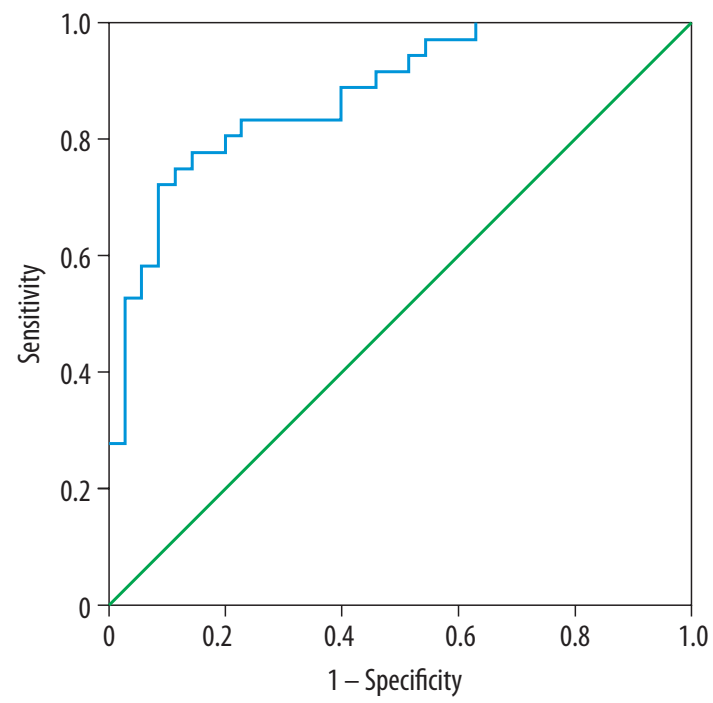

Figure 4. Receiver operating characteristic curve for malignancy index in identifying malignant thyroid nodules

and diagnostic accuracy were $81.5 \%$ (95\% CI: 69.2-89.7\%), 84.8\% (95\% CI: 71.0-92.8\%), and 83.1\% (95\% CI: 72.3$90.9 \%)$, respectively.

\section{Discussion}

Because of the high prevalence of thyroid nodules, especially in iodine-deficient areas, it is essential to find accurate and cost-effective ways of identifying high-risk thyroid nodules according to ultrasound features. By using advanced ultrasonographic devices, ultrasonography is now highly sensitive in determining the size and the number of the thyroid nodules. However, it still cannot reliably distinguish a malignant nodule from a benign one.

Ultrasonographic features such as hypoechogenicity, microcalcifications, intranodular vascularity, and the lack of halo margins are shown to be associated with the presence of malignancy in thyroid nodules [10-13]. However, these ultrasonographic features had relatively low sensitivity in identifying patients that indicate an invasive procedure such as FNAB [14-17].

Studies have shown that the nodules with a predominantly peri-nodular vascular pattern have a lower risk of malignancy than nodules with an exclusively central vascular pattern. Moon et al. [17] demonstrated that among ultrasonographic features of thyroid nodules, intra-nodular vascularity can predict the risk of malignancy more accurately than other ultrasonographic criteria. Ultrasound criteria, consisting of "taller than wide" shape, spiculated margin, marked hypoechogenicity, microcalcification, and macrocalcification, were reported to have a diagnostic accuracy of $77 \%$ in identifying malignant nodules when at least one US feature of malignancy was present [18]. The low accuracy observed in that study may be attributed to the absence of intra-nodular vascularity in the diagnostic criteria.

The PDUS-driven index of vascularity, a.k.a. malignancy index, had high performance in this study in differentiating malignant and benign thyroid nodules. Using a cut-off point of 0.092 , the malignancy index had a diagnostic accuracy of $83 \%$, and both the sensitivity and specificity were higher than the rates reported before in the literature for ultrasonographic parameters [18].

FNAB has been accepted as the gold standard test in the diagnosis of nodular thyroid disease, and FNAB under ultrasound guidance has a sensitivity rate of up to $100 \%$ in identifying malignant nodules [19]. Despite the high accuracy, the procedure is invasive and painful. PDUS-driven malignancy index can identify those with a higher risk of malignancy in thyroid nodules and can minimise the number of patients who require further testing with invasive FNAB.

There are a few limitations to mention here. A twodimensional PDUS approach was applied in this study because we wanted the diagnostic test to be feasible in almost every setting. Several studies have shown the advantages of using a 3D system because it visualises the whole lesion instead of applying limited transverse and longitudinal planes with two-dimensional ultrasound [20,21]. Testing this index in future in 3-D PDUS is recommended. Further prospective studies with larger sample sizes are warranted for external validation of this index.

\section{Conclusions}

The PDUS-driven malignancy index using contour-finding algorithm software was accurate and reliable in differentiating malignant and benign thyroid nodules. This method may be reliably applied as a pre-FNAB risk stratification tool to minimise the number of invasive procedures in nodular thyroid disease.

\section{Disclosure}

The authors declare no conflicts of interest. 


\section{References}

1. Singer PA, Cooper DS, Daniels GH, et al. Treatment guidelines for patients with thyroid nodules and well-differentiated thyroid cancer. American Thyroid Association. Arch Intern Med 1996; 156: 2165-2172.

2. Wong CK, Wheeler MH. Thyroid nodules: rational management. World J Surg 2000; 24: 934-941.

3. Hegedus L. Thyroid ultrasound. Endocrinol Metab Clin North Am 2001; 30: 339-360, viii-ix.

4. Park AY, Son EJ, Han K,et al. Shear wave elastography of thyroid nodules for the prediction of malignancy in a large scale study. Eur J Radiol 2015; 84: 407-412.

5. Hegedus L, Bonnema SJ, Bennedbaek FN. Management of simple nodular goiter: current status and future perspectives. Endocr Rev 2003; 24: 102-132.

6. Hong YJ, Son EJ, Kim EK, et al. Positive predictive values of sonographic features of solid thyroid nodule. Clin Imaging 2010; 34 : 127-133.

7. Mazzaglia PJ. Surgeon-performed ultrasound in patients referred for thyroid disease improves patient care by minimizing performance of unnecessary procedures and optimizing surgical treatment. World J Surg 2010; 34: 1164-1170.

8. Mendelson AA, Tamilia M, Rivera J, et al. Predictors of malignancy in preoperative nondiagnostic biopsies of the thyroid. J Otolaryngol Head Neck Surg 2009; 38: 395-400.

9. Park M, Shin JH, Han BK, et al. Sonography of thyroid nodules with peripheral calcifications. J Clin Ultrasound 2009; 37: 324-328.

10. Cappelli C, Castellano M, Pirola I, et al. The predictive value of ultrasound findings in the management of thyroid nodules. QJM 2007; 100: 29-35.

11. Horvath E, Majlis S, Rossi R, et al. An ultrasonogram reporting system for thyroid nodules stratifying cancer risk for clinical management. J Clin Endocrinol Metab 2009; 94: 1748-1751.
12. Salmaslioğlu A, Erbil Y, Dural C, et al. Predictive value of sonographic features in preoperative evaluation of malignant thyroid nodules in a multinodular goiter. World J Surg 2008; 32: 1948-1954.

13. Mohammadi A, Hajizadeh T. Evaluation of diagnostic efficacy of ultrasound scoring system to select thyroid nodules requiring fine needle aspiration biopsy. Int J Clin Exp Med 2013; 6: 641-648.

14. González-González A, Mate Valdezate A, Parra Arroyo A, et al. Diagnostic efficiency of sonographic findings of thyroid nodules in the detection of malignancy. Endocrinol Nutr 2010; 57: 240-244.

15. Leenhardt L, Tramalloni J, Aurengo H, et al. Echography of thyroid nodules. The echography specialist facing the clinician's requirements. Presse Med 1994; 23: 1389-1392.

16. Li QS, Chen S, Xiong H, et al. Papillary thyroid carcinoma on sonography. Clin Imaging 2010; 34: 121-126.

17. Moon HJ, Kwak JY, Kim MJ, et al. Can vascularity at power Doppler US help predict thyroid malignancy? Radiology 2010; 255: 260-269.

18. Moon WJ, Jung SL, Lee JH, et al. Benign and malignant thyroid nodules: US differentiation - multicenter retrospective study. Radiology 2008; 247: 762-770.

19. Abu-Salem OT. FNAB thyroid gland: comparison study between pre- and post-operative histological diagnosis. Arch Inst Pasteur Tunis 2003; 80: 57-60.

20. Li WB, Zhang B, Jiang YX, et al. Value of quantitative three-dimensional doppler ultrasound in the differentiation of benign and malignant thyroid nodules. Zhongguo Yi Xue Ke Xue Yuan Xue Bao 2015; 37: 300-304

21. Slapa RZ, Jakubowski WS, Slowinska-Srzednicka J, et al. Advantages and disadvantages of 3D ultrasound of thyroid nodules including thin slice volume rendering. Thyroid Res 2011; 4: 1. 